\title{
A Novel Nonrigid Registration Algorithm and Applications
}

\author{
J. Rexilius ${ }^{1}$, S.K. Warfield ${ }^{1}$, C.R.G. Guttmann ${ }^{1}$, X. Wei ${ }^{1}$, R. Benson ${ }^{2}$, L. Wolfson ${ }^{2}$, \\ M. Shenton ${ }^{1}$, H. Handels ${ }^{3}$, and R. Kikinis ${ }^{1}$ \\ 1 Surgical Planning Laboratory, Harvard Medical School \& Brigham and Women's Hospital, \\ 75 Francis St., Boston, MA 02115, USA. \\ rexilius@bwh.harvard.edu \\ ${ }^{2}$ Department of Neurology, University of Connecticut Health Center, USA. \\ 3 Institute for Medical Informatics, Medical University of Luebeck, Germany.
}

\begin{abstract}
In this paper we describe a new algorithm for nonrigid registration of brain images based on an elastically deformable model. The use of registration methods has become an important tool for computer-assisted diagnosis and surgery. Our goal was to improve analysis in various applications of neurology and neurosurgery by improving nonrigid registration.

A local gray level similarity measure is used to make an initial sparse displacement field estimate. The field is initially estimated at locations determined by local features, and then a linear elastic model is used to infer the volumetric deformation across the image. The associated partial differential equation is solved by a finite element approach. A model of empirically observed variability of the brain was created from a dataset of 154 young adults. Both homogeneous and inhomogeneous elasticity models were compared. The algorithm has been applied to medical applications including intraoperative images of neurosurgery showing brain shift and a study of gait and balance disorder.
\end{abstract}

\section{Introduction}

Developed about twenty years ago, nonrigid registration has meanwhile become a fundamental method for brain analysis in computer-assisted neurology and neurosurgery. An important issue thereby is the generation of deformation fields that reflect the transformation of an image in a realistic way with respect to the given anatomy. Due to lack of image structure, noise, intensity artifacts, computational complexity and a restricted time frame e.g. during surgery, it is not suitable to measure the deformation for each voxel. This leads to estimates of the deformation field only at sparse locations which have to be interpolated throughout the image.

In the last few years physically based elastic and viscous fluid models for nonrigid registration have become more and more popular [3] because they can constrain the underlying deformation in a plausible manner. However viscous fluid models [10], [11] have to be chosen carefully, since they allow large deformations which is not always suitable for medical applications concerning the brain. Furthermore, viscous fluid models driven by alignment of similar gray values may allow anatomically incorrect matches 
of different but adjacent structures through the same mechanism that allows large deformation matches. For example, one gyri may flow from the source brain to match two or more different gyri in a target brain, in a manner that may or may not be desirable.

In terms of physically based elastic models various algorithms have been described. Recent work (e.g. [7],[8]) proposed an active surface algorithm computed at the boundary of a regarded structure for an initial estimate of the deformation field. A drawback of this method is, that although it has been shown to be accurate close to the object's boundary, away from the boundaries the solution could potentially be less accurate. In [11] this idea was improved using statistical shape informations based on a set of images with hand-labeled points on the boundary of a structure which was included as an additional matching criterion. Even though such methods are promising for specific structures of the brain a robust $3 \mathrm{D}$ shape representation of the whole brain still remains difficult to achieve.

A different approach was proposed by Collins et.al. (see [6]). Their nonrigid registration algorithm was based on an iterative refinement of a local similarity measure using a simplex optimization. As this approach is constrained only by smoothing after correspondence estimation, the derived deformation field can only be accurate for specific regions of the brain. To achieve better results the method was improved by introducing various gyri and sulci of the brain as geometrical landmarks [5].

The aim of this paper is to present a new algorithm for computer-assisted neurology and neurosurgery. In order to get realistic deformations we propose an physically based elastic model, without requiring a segmentation or having the drawback that initial estimates of the deformation are only generated for the boundary of a considered structure. Therefore we used an enhanced approach based on the local similarity measure proposed by Collins et.al.. Furthermore we incorporated a model for inhomogeneous elasticities into our algorithm. The discretization of the underlying equation was done by a finite element technique, which has become a popular method for medical imaging applications (e.g. see [4] and [8]).

\section{Method}

The process of registration can be described as an optimization problem that minimizes both the difference between a template and a reference image and the deformation energy. We present a registration method, which basically runs in two steps. Based on a set of points extracted out of an image as described in (2.1), an initial sparse estimate of the deformation field is found by a local normalized cross-correlation (2.2). In a second step nonrigid registration is performed using an elastic model (2.3) which is constrained at the sparse estimates computed before.

\subsection{Feature Point Extraction}

Let $\Omega$ denote the domain of a volume $S: \Omega \rightarrow \mathbb{R}$ with voxel positions $\mathbf{x}=(x, y, z)^{\top}$, $\mathbf{x} \in \Omega$. In order to obtain suitable feature points for an initial sparse estimate of the deformation field, first the gradient magnitude is calculated out of blurred image intensities where only voxel higher than two standard deviations above the mean of the 
magnitude of the gradient are used for the correspondence detection (2.2). Figure 1 shows this process for one slice of a Magnetic Resonance (MR) scan of the brain.

To overcome the poor edge preserving properties of linear low-pass filters, we use a nonlinear diffusion filter which can be described as a solution of the partial differential equation (PDE)

$$
\partial_{t} S=\operatorname{div}\left(g\left(\left|\nabla S_{\sigma}\right|^{2}\right) \nabla S\right)
$$

with Neumann boundary conditions [15]. In order to reduce the noise sensitivity, the diffusion function $g: \mathbb{R} \rightarrow \mathbb{R}$ depends on the magnitude of the gradient of smoothed image intensities, computed by convolving $S$ with a Gaussian kernel of standard deviation $\sigma$. In our method, we use a diffusion function proposed by Weickert in [15]:

$$
g(x)=\left\{\begin{array}{ll}
1 & \text { for } x \leq 0 \\
1-\exp \left(\frac{-C}{(x / \lambda)^{4}}\right) & \text { for } x>0
\end{array} .\right.
$$

The parameter $\lambda$ separates regions of low contrast from those of high contrast. For the constant $C$ Weickert proposes $C=3.31448$ which gives visually good results and sets the flux $f(x)=x \cdot g(x)$ to an expected behavior, i.e. $f$ is increasing for values smaller or equal $\lambda$ and decreasing for values greater than $\lambda$. In our approach $\lambda$ was set interactively according to the considered volume. Furthermore for computational efficiency we use a parallel additive operator splitting (AOS) scheme which is stable for arbitrary large time steps and can be solved in linear time in terms of the image size. See [14] for details.

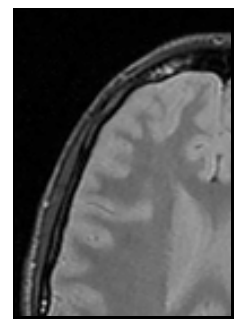

(a)

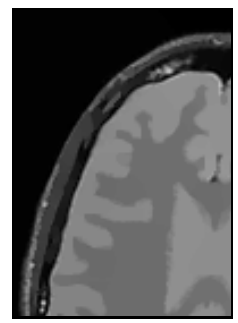

(b)

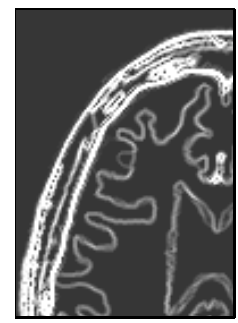

(c)

Fig. 1. Illustration of feature point extraction. For a better visual impression we only show a detail of the image. (a) Slice of MR scan; (b) Blurred image using a nonlinear diffusion filter; (c) Magnitude of the gradient of the blurred image after thresholding.

\subsection{Correspondence Detection}

After extracting feature points, the correspondence between the reference $R$ and template volume $T$ is computed for these points. We use the local normalized cross-correlation (NCC) as a similarity measure [6]

$$
\operatorname{NCC}(R, T, d)=\frac{\sum_{k \in \mathcal{N}(\mathbf{x})} f(R, k) \cdot f(T, d(k))}{\sqrt{\sum_{k \in \mathcal{N}(\mathbf{x})} f^{2}(R, k) \cdot \sum_{k \in \mathcal{N}(\mathbf{x})} f^{2}(T, d(k))}}, \forall \mathbf{x} \in \Omega,
$$


which is maximized in terms of the deformation function $d$ by a brute force search. The search space in our method is restricted to translations because other transformations like rotations or scaling would be of higher computational complexity. Assuming a window of size $(w \times w \times w)$, the local neighborhood of a voxel $\mathbf{x}$ is described by $\mathcal{N}(\mathbf{x}) \in\left\{(x-w, y-w, z-w)^{\top}, \ldots,(x+w, y+w, z+w)^{\top}\right\}$. The spatial points used for the NCC are computed by $f:(\mathbb{R}, \Omega) \rightarrow \mathbb{R}$, whose output $f(S, \mathbf{x})$ is given for voxels with high gradient magnitudes calculated out of blurred image intensities, as described in section (2.1).

\subsection{Interpolation from Sparse Displacement Estimates}

The sparse deformation estimates obtained for the feature points computed by a local NCC, are now introduced as external forces into an elastic model described in Equation (4). The underlying idea is to restrict the the registration process so that the resulting deformation field is a priori fixed by the estimates at these points.

For a three dimensional elastic body $\Omega$ the total potential energy is defined as the work of the internal strains minus the potential of the external forces and can be expressed as [18]:

$$
E(\mathbf{u})=\frac{1}{2} \int_{\Omega} \sigma^{\top} \epsilon d \Omega-\int_{\Omega} F^{\top} \mathbf{u} d \Omega,
$$

where the variables are given in terms of the strain vector, $\sigma$, the stress vector, $\epsilon$, the external forces, $F$ and the deformation field, $\mathbf{u}=(u(x, y, z), v(x, y, z), w(x, y, z))^{\top}$. We seek the deformation that minimizes the energy described in Equation (4). As we assume small deformations in terms of linear elasticities, the strain vector $\epsilon$ is given by

$$
\epsilon=\left(\frac{\partial u}{\partial x}, \frac{\partial v}{\partial y}, \frac{\partial w}{\partial z}, \frac{\partial u}{\partial y}+\frac{\partial v}{\partial x}, \frac{\partial v}{\partial z}+\frac{\partial w}{\partial y}, \frac{\partial w}{\partial x}+\frac{\partial u}{\partial z}\right)^{\top}
$$

The elastomechanical relation between stresses and strains can be expressed as

$$
\sigma=\left(\sigma_{x}, \sigma_{y}, \sigma_{z}, \tau_{x y}, \tau_{y z}, \tau_{z x}\right)^{\top}=D \epsilon
$$

with the elasticity matrix $D$. See [18] for the full details.

For the discretization we choose the finite element method (FEM), i.e. the domain $\Omega$ is approximated by a sum of elements. In our approach we use a regular mesh of tetrahedra. For every element the deformation $\mathbf{u}^{e}$ can now be described as a linear combination of so-called shape functions $N_{i}^{e}(x, y, z)=\frac{1}{6 V}\left(a_{i}+b_{i} x+c_{i} y+d_{i} z\right)$. A detailed computation for the volume $V$ of a tetrahedron and the coefficients is given in [18]. We assume a uniformly continuous transition between two elements, which leads to

$$
u^{e}(x, y, z)=\sum_{i=1}^{4} u_{i}^{e}(x, y, z) N_{i}^{e}(x, y, z) \quad, \forall e \in \Omega_{a},
$$

where $\Omega_{a}$ represents an approximation of the continuous domain $\Omega$.

A large system of equations can be computationally expensive to solve. To work against this, the resulting system of equations is solved in parallel with the Portable Extensible Toolkit for Scientific Computation (PETSc) package [2] in less than five 
minutes for a $256 \times 256 \times 124$ volume. Usually the NCC search is much more expensive than solving this system.

Typically elasticity parameters have been set arbitrarily and homogeneously [3]. Recently Lester et.al. [10] applied an inhomogeneous viscous fluid model to brain and neck registration using the manually segmented bone of the reference image as a region of high stiffness. Davatzikos et.al. [7] applied inhomogeneities to brain warping setting the elasticity parameters of the brain four times higher than their value in the ventricles.

Our approach differs in that the inhomogeneous elasticity parameters are derived from an empirical estimate of anatomical variability. We used a set of $154 \mathrm{MR}$ scans of the brain, first segmented into white matter, grey matter, cerebro-spinal fluid (CSF) and background using an EM-based statistical classification algorithm [16]. Then the head of each scan was aligned to an arbitrarily selected scan out of this database, using global affine transformations [13] and our nonrigid registration. In order to generate a model for inhomogeneous elasticities, we use a maximum-likelihood classification, where for each voxel the most likely structure and its frequency of occurrence at the voxel on all cases was stored. According to these results, the elasticity parameters are computed for every voxel. We choose a linear mapping for the computed frequency of occurrence of the identified brain tissues where the Poisson ratio $\nu$ was scaled in a range of $\nu \in[0.1,0.4]$ while Young's elasticity modulus $E$ has a range of $E \in[2000 k P a, 10000 k P a]$. The background was set to a low stiffness $E=1000 k P a$ and incompressibility parameter $\nu=0.05$, respectively. Figure 2 shows a slice of the computed model and the associated intensities for $\nu$.

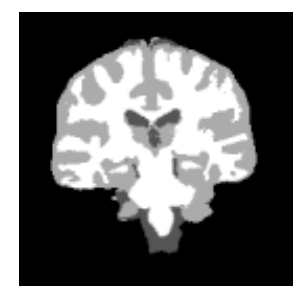

(a)

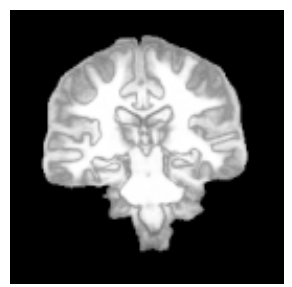

(b)

Fig. 2. Computed model of empirically observed variability. (a) Slice of the model after maximum-likelihood classification; (b) Computed incompressibility parameter (Poisson ratio $\nu$ ) for each voxel of the same slice. Dark regions imply a low value for $\nu$.

\section{Experimental Results}

To evaluate the two different methods presented in this paper, we show some results obtained for varying medical applications. In case of homogeneous elasticities we use $E=3000 k P a$ for the Young elasticity modulus, and $\nu=0.4$ for the Poisson ratio, as used by Ferrant et. al. [8]. 


\subsection{Illustration of Nonrigid Registration with Homogeneous and Inhomogeneous Elasticities}

In order to show the behavior of a deformation model with homogeneous and inhomogeneous elasticities, the algorithm was applied to register 159 MR scans of the brain of young adults. Therefore each scan was first globally registered to an arbitrarily chosen dataset by an affine transformation [13]. The nonrigid registration with homogeneous and inhomogeneous elasticities was then applied to the aligned data (Figure 3).

An analysis of the summed squared differences showed an improvement of $2 \%$ using inhomogeneous elasticities. This rather small effect is due to the setting of feature points in our experiments. As it can be seen in Figure 2 large regions of white matter only have a small range of anatomical variability, i.e. large number of fixed deformation estimates constrain the interpolation done by the elastic model. Further research will investigate new approximation schemes to address this.

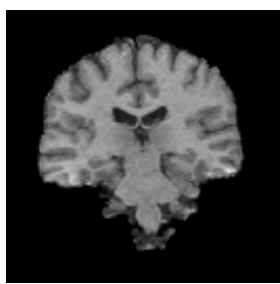

(a)

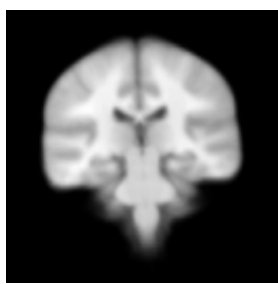

(b)

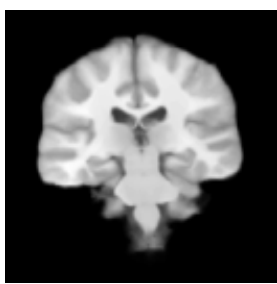

(c)

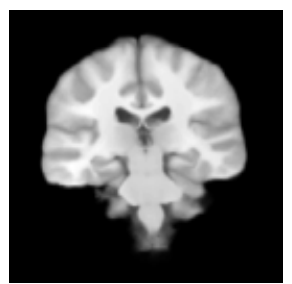

(d)

Fig. 3. Results of study applying rigid and nonrigid registration to 159 subjects. (a) Slice of reference volume; (b)-(d) Result after registration and averaging over all scans using: (b) affine registration; (c) nonrigid registration with homogeneous elasticities; (d) nonrigid registration with inhomogeneous elasticities.

\subsection{Capturing Brain Shift}

During neurosurgery the shape of the brain changes which can be considered as nonrigid deformation. Recently a fast biomechanical simulation of this brain shift was proposed in [12]. This algorithm used segmentation to identify key structures and to remove noise and intensity artifacts. In our new method we can derive sparse estimates without a segmentation so long as noise and intensity artifacts are minimal. This allows estimates to be obtained in a larger region of the brain. Figure 4 shows images of the brain before (Fig. 4(a)) and after craniotomy (Fig. 4(b)) as well as the reference image after craniotomy with overlayed initial (Fig. 4 (c)) and deformed image (Fig. 4 (d)). It can be observed, that the brain shift was successfully captured. Even though the results show some artifacts in the area of the brain surface. This is due to a massive change in the patients brain structure during craniotomy resulting in a large cavity which cannot be computed with an elastic model out of the initial image. As this area only contains 
cerebro-spinal fluid we haven't addressed this issue in our current studies. More validation experiments will be required to appropriately assess the potential for this method. These are currently under way.

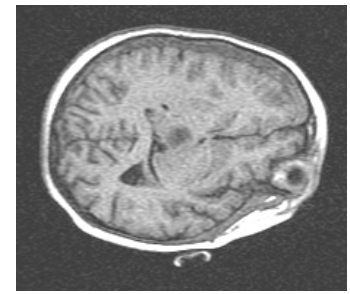

(a)

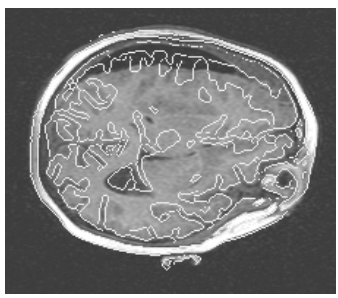

(c)

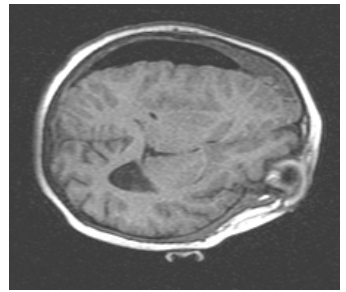

(b)

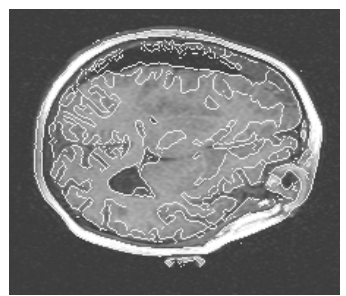

(d)

Fig.4. Elastic matching applied to MR scan of the brain obtained during neurosurgery. (a) Image at the beginning of the surgery; (b) Image after craniotomy; (c) Slice of reference volume with overlayed contours of initial scan; (d) Slice of reference volume with overlayed contours of deformed volume;

\subsection{Analysis of Balance and Gait Disorders}

Impaired mobility caused by gait and balance disorders is common in older adults. Central nervous system disease, as revealed by white matter signal abnormalities (WMSAs) observed by MRI, is believed to play a significant role in gait disorder while still being common in asymptomatic elderly subjects [9]. Therefore it is interesting to understand the spatial distribution of WMSAs in impaired and asymptomatic subjects. The alignment of impaired mobility subjects and controls in a common coordinate system can help to determine the spatial distribution of such WMSAs. A commonly applied but relatively low order nonrigid registration algorithm is that provided by SPM [17],[1]. We wished to investigate the use of our high dimensional nonrigid registration to determine spatial distribution of WMSAs associated with gait disorder by comparing the spatial distribution of WMSAs between two groups - 16 subjects with impaired mobility, and 12 age matched control subjects without impaired mobility.

Figure 5 (a) shows the slice of one subject used as reference image. The results of the nonrigid registration after averaging over all scans are presented in Figure 5(b). Figure 5 (c) illustrates the results of a Chi-square test, with values significant at the $\alpha=0.01$ 
level shown as bright pixels, and with lower values scaled linearly in intensity to zero (black). The results show clearly that people with balance and gait disorders have a statistically significant increase in lesions in two specific regions of the white matter of the brain, as compared to asymptomatic elderly subjects.

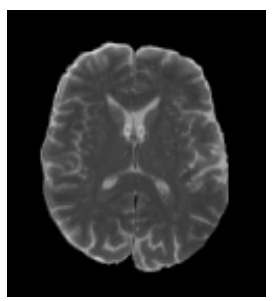

(a)

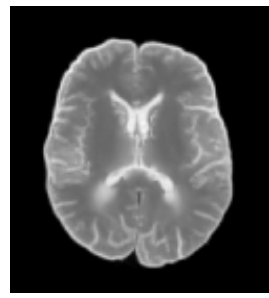

(b)

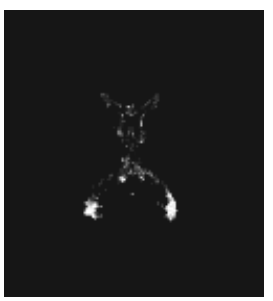

(c)

Fig. 5. Results of a study analyzing balance and gait disorders. (a) Slice of reference volume; (b) Averaged cases of the study after nonrigid registration using inhomogeneous elasticities; (c) Result of a voxel independent chi-square statistic.

\section{Discussion and Conclusion}

We have presented a method for nonrigid registration which was used for various applications in neurology and neurosurgery including simulation of brain shift and a study of balance and gait disorders. The applied elastic model provides us thereby with the ability to simulate realistic deformation applying inhomogeneous elasticities derived by a model of empirically observed variability. A local similarity measure was used to constrain the model which was discretized by the finite element method.

Further work will investigate alternative similarity measures and features extractions, e.g. local structure features. We also plan to enhance this approach incorporating the anisotropy of certain brain tissue structures.

Acknowledgements: This investigation was supported by NIH P41 RR13218, NIH P01 CA67165 and NIH R01 RR11747.

\section{References}

1. J. Ashburner and K.J. Friston: Spatial Normalization. In Brain Warping. Ed. Arthur W. Toga, (Academic Press) Ch.2:27-44, 1999.

2. S. Balay, W.D. Gropp, L.C. McInnes and B.F. Smith: PETSc 2.0 users manual. Tech. Rep. ANL-95/11 - Revision 2.0.28, Argonne National Laboratory, 2000

3. R. Bajcsy and S. Kovacic: Multiresolution Elastic Matching. Computer Vison, Graphics and Image Processing, 46:1-21, 1989.

4. M. Bro-Nielsen: Finite element modeling in medical VR. Journal of the IEEE, 86(3):490-503, 1998. 
5. D.L. Collins, G. Le Goualher and A.C. Evans: Non-linear Cerebral Registration with Sulcal Constrains. In MICCAI 1998, Cambridge, MA, USA, 1998 pages 974-984.

6. D.L. Collins: 3D Model-based segmentation of individual brain structures for magnetic resonance imaging data. $\mathrm{PhD}$ thesis, 1994.

7. C. Davatzikos: Spatial Transformation and Registration of Brain Images Using Elastically Deformable Models. Comp. Vis. and Image Understanding, Special Issue on Medical Imaging, 66(2):207-222, May 1997

8. M. Ferrant, S.K. Warfield, A. Nabavi, F.A. Jolesz and R. Kikinis: Registration of 3D Intraoperative MR Images of the Brain Using a Finite Element Biomechanical Model. In MICCAI 2000, Pittsburgh, Pennsylvania, USA, 2000, pages 19-28.

9. C.R.G. Guttmann, R. Benson, S.K. Warfield, X. Wei, M.C. Anderson, C. Hall, K. AbuHasaballah, J.P. Mugler and L. Wolfson: White matter abnormalities in mobility-impaired older persons. Neurology, 54:1277-1283, 2000

10. H. Lester, S.R. Arridge, K.M. Jansons, L. Lemieux, J.V. Hajnal and A. Oatridge: Non-linear Registration with the Variable Viscosity fluid Algorithm. In IPMI 1999, pages 238-251, 1999.

11. Y. Wang, L.H. Staib: Physical model-based non-rigid registration incorporating statistical shape information. Medical Image Analysis, 4(2000) pages 7-20, 2000.

12. S.K. Warfield, M. Ferrant, X. Gallez, A. Nabavi, F.A. Jolesz and R. Kikinis: Real-Time biomechanical Simulation of Volumetric Brain Deformation for Image Guided Neurosurgery. High Performance Networking and Computing Conference, Dallas, USA, 230:1-16, 2000.

13. S.K. Warfield, F.A. Jolesz and R. Kikinis: A High Performance Computing Approach to the Registration of Medical Imaging Data. Parallel Computing 24:1345-1368, 1998.

14. J. Weickert, B. ter Haar Romeny, M.A. Viergever: Efficient and reliable schemes for nonlinear diffusion filtering. IEEE Trans. Image Processing, Vol. 7, pages 398-410, March 1998.

15. J. Weickert: Anisotropic diffusion in image processing. Teubner Verlag, Stuttgart, 1997.

16. W.M. Wells, R. Kikinis, W.E.L. Grimson, F. Jolesz: Adaptive segmentation of MRI data. IEEE Transactions on Medical Imaging, 15:429-442, 1996

17. K.J. Worsley, S. Marrett, P. Neelin, A.C. Vandal, J.J. Friston and A.C. Evans: A unified Statistical Approach for Determining Significant Signals in Images of Cerebral Activation In Human Brain Mapping, pages 58-73, 1996

18. O.C. Zienkewickz, R.L. Taylor: The Finite Element Method. McGraw Hill Book Co., 1987. 